\title{
Randomized Dividends in a Discrete Insurance Risk Model with Stochastic Premium Income
}

\author{
Wenguang $Y u^{1,2}$ \\ ${ }^{1}$ School of Mathematics, Shandong University, Jinan 250100, China \\ ${ }^{2}$ School of Insurance, Shandong University of Finance and Economics, Jinan 250014, China
}

Correspondence should be addressed to Wenguang Yu; yuwg@mail.sdu.edu.cn

Received 3 December 2012; Accepted 23 January 2013

Academic Editor: Guangchen Wang

Copyright (C) 2013 Wenguang Yu. This is an open access article distributed under the Creative Commons Attribution License, which permits unrestricted use, distribution, and reproduction in any medium, provided the original work is properly cited.

The compound binomial insurance risk model is extended to the case where the premium income process, based on a binomial process, is no longer a constant premium rate of 1 per period and insurer pays a dividend of 1 with a probability $q_{0}$ when the surplus is greater than or equal to a nonnegative integer $b$. The recursion formulas for expected discounted penalty function are derived. As applications, we present the recursion formulas for the ruin probability, the probability function of the surplus prior to the ruin time, and the severity of ruin. Finally, numerical example is also given to illustrate the effect of the related parameters on the ruin probability.

\section{Introduction}

The classical compound binomial insurance risk model is a discrete time risk process with the following features. The premium received in each period is 1 . The number of claims is regulated by a binomial process $N(t), t=0,1,2, \ldots$ In any time period, the probability of a claim is $p(0<p<1)$, and the probability of no claim is $q=1-p$. We assume that claims occur at the end of the period and denote by $\xi_{t}=1$ the event where a claim occurs in the time period $(t-1, t]$ and denote by $\xi_{t}=0$ the event where no claim occurs in the time period $(t-1, t]$. Then $N(t)=\sum_{k=1}^{t} \xi_{k}$ for $t \geq 1$ and $N(0)=0$. The individual claim amounts $X_{1}, X_{2}, X_{3}, \ldots$ are independent, identically distributed (i.i.d.) positive integervalued random variables with distribution $F(x)=1-\bar{F}(x)=$ $\operatorname{Pr}(X \leq x)$ and probability function $f(x)$ with finite mean $\mu$. They are independent of the binomial process $\{N(t)\}$. For $t=1,2, \ldots$, the surplus at time $t$ is

$$
U(t)=u+t-\sum_{k=1}^{t} X_{k} \xi_{k},
$$

where $U(0)=u>0$ is the initial surplus. The risk model (1) was first proposed by Gerber [1] and has been further studied by many authors during the last few years. See, for example, Shiu [2], Willmot [3], and Dickson [4].

Recently, Gerber and Shiu [5] introduced a discounted penalty function with respect to the time of ruin, the surplus immediately before ruin, and the deficit at ruin, which has been proven to be a powerful analytical tool. Some related results can be found in Cheng et al. [6], Lin et al. [7], Wang and Wu [8], Wang et al. [9], and Willmot and Wood [10]. For the compound binomial model, Tan and Yang [11] consider the risk model (1) modified by the inclusion of dividends and derive recursion formulas and an asymptotic estimate for the ruin probability, the probability function of the surplus prior to the ruin time, and the severity of ruin, and so forth. Landriault [12] considers the compound binomial model with a multithreshold dividend structure and randomized dividend payments. Using the roots of a generalization of Lundberg's fundamental equation and the general theory of difference equations, he derives an explicit expression for the Gerber-Shiu discounted penalty function with any initial surplus. This result generalizes the main result of Tan and Yang [11]. Fang et al. [13] extend the risk model (1) to the case where the premium income process, based on a binomial process, is no longer a linear function and examine the expected discounted value of a penalty at ruin, which is 
considered as a function of the initial surplus. Li [14] studies the moments of the present value of total dividend payments in the compound binomial risk model in the presence of a constant dividend barrier and stochastic interest rates. Some recent developments about compound binomial model can be found in Yuen and Guo [15], Liu and Zhao [16], Yu et al. [17], Hao and Yang [18], Tan and Yang [19], and Bao and Liu [20].

In this paper, motivated by the work of Tan and Yang [11] and Fang et al. [13], we consider the compound binomial model (1) modified by the inclusion of dividends and the stochastic premium income. We assume that the stochastic premiums are received at the beginning of each time period. It is no longer a constant premium rate of 1 per period but another binomial process with parameter $p_{1}\left(0 \leq p_{1}<1\right)$, independent of $\{N(t), t=0,1,2, \ldots\}$ and $\left\{X_{k}, k=1,2, \ldots\right\}$. In addition, we suppose that the insurer will pay a dividend of 1 with a probability $q_{0}\left(0 \leq q_{0}<1\right)$ in each time period if the surplus is greater than or equal to a nonnegative integer $b$ at the beginning of the period. It implies that the randomized dividend payments will only possibly occur at the beginning of each period, right after receiving the stochastic premium payment. We will derive the recursion formulas for the expected discounted penalty function, the ruin probability, the probability function of the surplus prior to the ruin time and the severity of ruin.

The rest of the paper proceeds as follows. In Section 2, the risk model is introduced. In Section 3, the recursion formulas for the expected discounted penalty function associated with the time of ruin when the discount factor $v=1$ are derived. As applications, in Section 4, we give the recursion formulas for the ruin probability, the probability function of the surplus prior to the ruin time, and the severity of ruin. Finally, a numerical example is also given to illustrate the effect of parameters on the ruin probability.

\section{The Risk Model}

In this section, we consider a discrete time risk process based on the compound binomial model, which is the extension of the work of Tan and Yang [11]. The surplus process is given by

$$
U(t)=u+M(t)-Z_{t}-S_{t}, \quad t=0,1,2, \ldots,
$$

where $S_{t}$ is defined as $\sum_{k=1}^{t} X_{k} \xi_{k}$, for $t \geq 1$ and $S_{0}=0$. The binomial process $\{M(t), t=0,1,2, \ldots\}$ with parameter $p_{1}$ is corresponding to the number of the customers up to time $t$. We denote by $\zeta_{k}=1$ the event where a payment occurs in period $(t-1, t]$ denote by and $\zeta_{k}=0$ the event where no payment occurs in period $(t-1, t]$. If the event occurs in period $(t-1, t]$, we suppose that the event happen at the beginning of the period. Let $\operatorname{Pr}\left(\zeta_{k}=1\right)=p_{1}, \operatorname{Pr}\left(\zeta_{k}=0\right)=$ $q_{1}=1-p_{1} . Z_{t}$ is defined as

$$
Z_{t}=\sum_{k=1}^{t} \eta_{k} I(U(k-1) \geq b),
$$

for $t>1$ and $Z_{0}=0 . b$ is a fixed nonnegative integer, $I(A)$ is the indicator function of a set $A$, and $\eta_{k}(k \geq 1)$ is a series of randomized decision functions that are mutually independent, identically distributed, and independent of $\sum_{k=1}^{t} X_{k} \xi_{k}$. In detail, we denote by $\eta_{k}=1$ the event where a dividend of 1 is paid at the time $k$ and denote by $\eta_{k}=0$ the event where no dividend is paid at the time $k$. Assume $\operatorname{Pr}\left(\eta_{k}=1\right)=q_{0}, \operatorname{Pr}\left(\eta_{k}=0\right)=p_{0}=1-q_{0}$. Then (2) is equivalent to

$$
\begin{aligned}
U(t)= & u+\sum_{k=1}^{t} \zeta_{k}-\sum_{k=1}^{t} \eta_{k} I(U(k-1) \geq b) \\
& -\sum_{k=1}^{N(t)} X_{k}=u-V(t), \quad t=0,1,2, \ldots
\end{aligned}
$$

It is reasonable that the randomized decision functions $\eta_{k}(k \geq 1)$ are brought in to decide the periods with a dividend. This can be interpreted as follows: the return on the investment by an insurer in each time period can be regarded as being stochastic, and if the return on the investment in the present period is greater than a given level $b$, then the insurer will pay a benefit of 1 to the insured. But, in model (2), if the surplus is smaller than $b$ at the beginning of the present period, then a decision for paying a benefit of 1 will be cancelled. Certainly, the randomized decisions can also be related to some other things that occur with probability $q_{0}$ and do not occur with probability $p_{0}$ in each time period, for example, natural disasters. The model (4) is a sort of generalization of the classic risk model and is exactly the risk model [13] if $q_{0}=0$. Hence our results in this paper include the corresponding results of the risk model [13].

In this paper, we always assume that the positive security loading condition holds; that is, if we denote by $\theta$ the relative security loading, then

$$
\theta=\frac{p_{1}-q_{0}}{p \mu}-1
$$

Let $p(k)=\operatorname{Pr}(X=k), k=1,2, \ldots$, be the common probability function of the claim amounts. (The value of $p(k)$ is zero if $k$ is not a positive integer.)

Let

$$
\begin{gathered}
p(0)=0, \quad P(n)=\sum_{k=1}^{n} p(k), \quad \forall n \geq 1, \\
\bar{P}(n)=1-P(n), \quad \forall n \geq 0, \quad P(0)=0, \\
\mu=E[X]=\sum_{n=0}^{\infty} n p(n)=\sum_{n=0}^{\infty} \bar{P}(n) .
\end{gathered}
$$

Define

$$
T=\inf \{t \geq 1: U(t)<0\} \quad(\inf \emptyset=\infty)
$$

to be the time of ruin. Define the ultimate ruin probability

$$
\psi(u)=\operatorname{Pr}(T<\infty \mid U(0)=u) .
$$

Except for the ruin probability, other important ruin quantities in ruin theory include the generating function of 
the time of ruin, $E\left(r^{T}\right)$; the surplus before ruin, denoted by $U_{T-1}$; and the deficit at ruin, $\left|U_{T}\right|$. A unified method to study these ruin quantities is to consider the (expected discounted) penalty function associated with the time of ruin by defining

$$
m_{v}(u)=E\left[v^{T} w\left(U_{T-1},\left|U_{T}\right|\right) I(T<\infty) \mid U(0)=u\right]
$$

where $w(x, y), x \geq 0, y \geq 0$, is a nonnegative bounded function; $0<v \leq 1$ is the discount factor.

In this paper, we only obtain the recursion formula for $m_{1}(u)$, that is, the discount factor $v=1$. However, this is enough for us to study some important ruin quantities except for $E\left(r^{T}\right)$. Let $m_{1}(u)=m(u)$. In this paper, we adopt the convention that $\sum_{a}^{d}=0$ when $d<a$.

\section{Recursive Formulas for the Expected Discounted Penalty Function}

In this section, we derive the renewal equations for the expected discounted penalty function $m(u)$.

Theorem 1. (i) For all $u \geq b$, the penalty function $m(u)$ satisfies

$$
\begin{aligned}
m(u+1) & \\
= & \frac{1-q q_{1} p_{0}-q p_{1} q_{0}}{q p_{1} p_{0}} m(u)-\frac{q_{1} q_{0}}{p_{1} p_{0}} m(u-1) \\
& -\frac{p q_{1}}{q p_{1} p_{0}} \sum_{k=0}^{u} m(k)\left[p_{0} p(u-k)+q_{0} p(u-1-k)\right] \\
& -\frac{p q_{1}}{q p_{1} p_{0}} \sum_{k=u+1}^{\infty} w(u, k-u)\left[p_{0} p(k)+q_{0} p(k-1)\right] \\
& -\frac{p}{q p_{0}} \sum_{k=0}^{u} m(k)\left[p_{0} p(u+1-k)+q_{0} p(u-k)\right] \\
& -\frac{p}{q p_{0}} \sum_{k=u+1}^{\infty} w(u, k-u)\left[p_{0} p(k+1)+q_{0} p(k)\right]
\end{aligned}
$$

$$
\begin{aligned}
& q p_{1} p_{0} m(u+1)-q q_{1} q_{0} m(u) \\
& =p q_{1} \sum_{k=0}^{u} m(k)\left[p_{0} \bar{P}(u-k)+q_{0} \bar{P}(u-1-k)\right] \\
& \quad+p p_{1} \sum_{k=0}^{u} m(k)\left[p_{0} \bar{P}(u+1-k)+q_{0} \bar{P}(u-k)\right] \\
& \quad+p q_{1} \sum_{k=u+1}^{\infty} \sum_{i=k+1}^{\infty} w(k, i-k)\left[p_{0} p(i)+q_{0} p(i-1)\right] \\
& \quad+p p_{1} \sum_{k=u+1}^{\infty} \sum_{i=k+1}^{\infty} w(k, i-k)\left[p_{0} p(i+1)+q_{0} p(i)\right] .
\end{aligned}
$$

(ii) If $b \geq 1$, then $m(0), m(1), \ldots, m(b)$ satisfy the following linear equations:

$$
\begin{gathered}
p_{1} q m(0)-q_{0} m(b-1)=\delta, \\
q p_{1} m(u+1)+\left(q q_{1}+p p_{1} p(1)-1\right) m(u) \\
+p q_{1} \sum_{k=0}^{u-1} m(k) p(u-k) \\
+p p_{1} \sum_{k=0}^{u-1} m(k) p(u+1-k) \\
=f(u+1), \quad(u=0,1,2, \ldots, b-1),
\end{gathered}
$$

where

$$
\begin{aligned}
f(u+1)= & -p q_{1} \sum_{k=u+1}^{\infty} w(u, k-u) p(k) \\
& -p p_{1} \sum_{k=u+2}^{\infty} w(u, k-u-1) p(k), \\
\delta= & p p_{0} \sum_{k=0}^{b-1} \sum_{i=k+1}^{\infty} w(k, i-k)\left[p_{1} p(i+1)+q_{1} p(i)\right] \\
& +p q_{0} \sum_{k=0}^{b-2} \sum_{i=k+1}^{\infty} w(k, i-k)\left[p_{1} p(i+1)+q_{1} p(i)\right] \\
& +p q_{1} \sum_{k=b}^{\infty} \sum_{i=k+1}^{\infty} w(k, i-k)\left[p_{0} p(i)+q_{0} p(i-1)\right] \\
& +p p_{1} \sum_{k=b}^{\infty} \sum_{i=k+1}^{\infty} w(k, i-k)\left[p_{0} p(i+1)+q_{0} p(i)\right] .
\end{aligned}
$$

Proof. We consider $U(t)$ in the first time period $(0,1]$ and separate the eight possible cases as follows.

(1) No claim occurs in $(0,1]$, no premium arrives in $(0,1]$ and paying no dividend in $(0,1]$.

(2) No claim occurs in $(0,1]$, no premium arrives in $(0,1]$ and paying a dividend of 1 in $(0,1]$ (if $u<b$, this case does not exist).

(3) No claim occurs in $(0,1]$, a premium arrives in $(0,1]$ and paying no dividend in $(0,1]$.

(4) A claim occurs in $(0,1]$, no premium arrives in $(0,1]$ and paying no dividend in $(0,1]$.

(5) A claim occurs in $(0,1]$, a premium arrives in $(0,1]$ and paying no dividend in $(0,1]$.

(6) A claim occurs in $(0,1]$, no premium arrives in $(0,1]$ and paying a dividend of 1 in $(0,1]$ (if $u<b$, this case does not exist).

(7) No claim occurs in $(0,1]$, a premium arrives in $(0,1]$ and paying a dividend of 1 in $(0,1]$ (if $u<b$, this case does not exist). 
(8) A claim occurs in $(0,1]$, a premium arrives in $(0,1]$ and paying a dividend of 1 in $(0,1]$ (if $u<b$, this case does not exist).

Considering whether $u<b$ or $u \geq b$ and using the laws of conditional probability, the penalty function is equal to

$$
\begin{aligned}
& m(u)=q q_{1} m(u)+q p_{1} m(u+1) \\
& +p q_{1} \sum_{k=1}^{u} m(u-k) p(k) \\
& +p q_{1} \sum_{k=u+1}^{\infty} w(u, k-u) p(k) \\
& +p p_{1} \sum_{k=1}^{u+1} m(u+1-k) p(k) \\
& +p p_{1} \sum_{k=u+2}^{\infty} w(u, k-u-1) p(k), \\
& \forall 0 \leq u<b, \\
& m(u)=q q_{1} p_{0} m(u)+q q_{1} q_{0} m(u-1) \\
& +q p_{1} p_{0} m(u+1)+q p_{1} q_{0} m(u) \\
& +p q_{1} p_{0} \sum_{k=1}^{u} m(u-k) p(k) \\
& +p q_{1} p_{0} \sum_{k=u+1}^{\infty} w(u, k-u) p(k) \\
& +p p_{1} p_{0} \sum_{k=1}^{u+1} m(u+1-k) p(k) \\
& +p p_{1} p_{0} \sum_{k=u+2}^{\infty} w(u, k-u-1) p(k) \\
& +p q_{1} q_{0} \sum_{k=1}^{u-1} m(u-1-k) p(k) \\
& +p q_{1} q_{0} \sum_{k=u}^{\infty} w(u, k-u+1) p(k) \\
& +p p_{1} q_{0} \sum_{k=1}^{u} m(u-k) p(k) \\
& +p p_{1} q_{0} \sum_{k=u+1}^{\infty} w(u, k-u) p(k),
\end{aligned}
$$$$
\forall u \geq b .
$$

When $t \geq b$, summing (16) over $u$ from $b$ to $t$, we obtain

$$
\begin{aligned}
& q p_{1} p_{0}[m(t+1)-m(b)]-q q_{1} q_{0}[m(t)-m(b-1)] \\
& =p q_{1} \sum_{k=0}^{t} m(k)\left[p_{0} \bar{P}(t-k)+q_{0} \bar{P}(t-1-k)\right] \\
& -p q_{1} \sum_{k=0}^{b-1} m(k)\left[p_{0} \bar{P}(x-1-k)+q_{0} \bar{P}(x-k-2)\right] \\
& -p q_{1} \sum_{k=b}^{t} \sum_{i=k+1}^{\infty} w(k, i-k)\left[p_{0} p(i)+q_{0} p(i-1)\right] \\
& +p p_{1} \sum_{k=0}^{t} m(k)\left[p_{0} \bar{P}(t+1-k)+q_{0} \bar{P}(t-k)\right] \\
& -p p_{1} \sum_{k=0}^{b-1} m(k)\left[p_{0} \bar{P}(x-k)+q_{0} \bar{P}(x-1-k)\right] \\
& -p p_{1} \sum_{k=b}^{t} \sum_{i=k+1}^{\infty} w(k, i-k)\left[p_{0} p(i+1)+q_{0} p(i)\right], \\
& \forall t \geq b .
\end{aligned}
$$

Note that $w(x, y)$ is defined as a bounded function. We define $\|w\|=\sup \{w(x, y)\}$ again; then $\|w\|<\infty$. According to the definition of $m(u)$, we know that

$$
|m(u)| \leq\|w\| \operatorname{Pr}(T<\infty \mid U(0)=u)=\|w\| \psi(u)
$$

Because the relative security loading $\theta>0$ and $\lim _{u \rightarrow \infty} \psi(u)=0$, thus

$$
\lim _{u \rightarrow \infty} m(u)=0
$$

By the dominated convergence theorem and (19), and letting $t \rightarrow \infty$ in (17), we get

$$
\begin{aligned}
& -q p_{1} p_{0} m(b)+q q_{1} q_{0} m(b-1) \\
& =-p q_{1} \sum_{k=0}^{b-1} m(k)\left[p_{0} \bar{P}(x-1-k)+q_{0} \bar{P}(x-k-2)\right] \\
& -p q_{1} \sum_{k=b}^{\infty} \sum_{i=k+1}^{\infty} w(k, i-k)\left[p_{0} p(i)+q_{0} p(i-1)\right] \\
& -p p_{1} \sum_{k=0}^{b-1} m(k)\left[p_{0} \bar{P}(x-k)+q_{0} \bar{P}(x-1-k)\right] \\
& -p p_{1} \sum_{k=b}^{\infty} \sum_{i=k+1}^{\infty} w(k, i-k)\left[p_{0} p(i+1)+q_{0} p(i)\right] .
\end{aligned}
$$

Plugging (20) into (17) and rearranging terms we obtain (11). 
If $b \geq 1$ and $t<b$, summing (15) over $u$ from 0 to $t$, we get

$$
\begin{array}{r}
p_{1} q[m(t+1)-m(0)] \\
=p \sum_{k=0}^{t} m(k)\left[p_{1} \bar{P}(t+1-k)+q_{1} \bar{P}(t-k)\right] \\
-p \sum_{k=0}^{t} \sum_{i=k+1}^{\infty} w(k, i-k)\left[p_{1} p(i+1)+q_{1} p(i)\right], \\
\forall 0 \leq t<b .
\end{array}
$$

Replacing $t$ by $t-1$ in (21) and considering $\sum_{a}^{d}=0$ when $d<a$ yield

$$
\begin{array}{r}
m(t)-q_{1} q m(t)-p_{1} q m(0) \\
=p \sum_{k=0}^{t} m(k)\left[p_{1} \bar{P}(t-k)+q_{1} \bar{P}(t-1-k)\right] \\
-p \sum_{k=0}^{t-1} \sum_{i=k+1}^{\infty} w(k, i-k)\left[p_{1} p(i+1)+q_{1} p(i)\right], \\
\forall 0 \leq t<b .
\end{array}
$$

Multiplying (21) by $p_{0}$, multiplying (22) by $q_{0}$, and summing yield

$$
\begin{array}{r}
p_{0} p_{1} q m(t+1)+q_{0}\left(1-q_{1} q\right) m(t)-p_{1} q m(0) \\
=p p_{0} \sum_{k=0}^{t} m(k)\left[p_{1} \bar{P}(t+1-k)+q_{1} \bar{P}(t-k)\right] \\
\quad-p p_{0} \sum_{k=0}^{t} \sum_{i=k+1}^{\infty} w(k, i-k)\left[p_{1} p(i+1)+q_{1} p(i)\right] \\
+p q_{0} \sum_{k=0}^{t} m(k)\left[p_{1} \bar{P}(t-k)+q_{1} \bar{P}(t-1-k)\right] \\
-p q_{0} \sum_{k=0}^{t-1} \sum_{i=k+1}^{\infty} w(k, i-k)\left[p_{1} p(i+1)+q_{1} p(i)\right], \\
\forall 0 \leq t<b .
\end{array}
$$

If $t=b-1$, from (23) and (20) we get (12). Equation (13) comes directly from (15).

\section{Application}

4.1. Some Ruin Quantities. In this section, we give some examples of ruin quantities to illustrate applications of the recursive formulas derived in Section 3.
Example 2. Letting $w(x, y)=1$ and $v=1$, we have $m(u)=$ $E[I(T<\infty)]=\psi(u)$. In this case, $f(u+1)=-p\left[q_{1} \bar{P}(u)+\right.$ $\left.p_{1} \bar{P}(u+1)\right], \delta=p\left(\mu-p_{1}\right)$. Thus, by Theorem $1, \psi(u)$ satisfies

$$
\psi(u+1)
$$

$$
\begin{aligned}
= & \frac{1-q q_{1} p_{0}-q p_{1} q_{0}}{q p_{1} p_{0}} \psi(u) \\
& -\frac{q_{1} q_{0}}{p_{1} p_{0}} \psi(u-1) \\
& -\frac{p q_{1}}{q p_{1} p_{0}} \sum_{k=0}^{u-1} \psi(k)\left[p_{0} p(u-k)+q_{0} p(u-1-k)\right] \\
& -\frac{p q_{1}}{q p_{1} p_{0}}\left[p_{0} \bar{P}(u)+q_{0} \bar{P}(u-1)\right] \\
& -\frac{p}{q p_{0}} \sum_{k=0}^{u} \psi(k)\left[p_{0} p(u+1-k)+q_{0} p(u-k)\right] \\
& -\frac{p}{q p_{0}}\left[p_{0} \bar{P}(u+1)+q_{0} \bar{P}(u)\right] \quad \forall u \geq b,
\end{aligned}
$$

which can be rewritten as

$$
\begin{aligned}
\psi(u+1) & \\
= & \frac{q_{0} q_{1}}{p_{0} p_{1}} \psi(u) \\
& +\frac{p q_{1}}{p_{0} p_{1} q} \sum_{k=0}^{u} \psi(k)\left[p_{0} \bar{P}(u-k)+q_{0} \bar{P}(u-1-k)\right] \\
& +\frac{p}{p_{0} q} \sum_{k=0}^{u} \psi(k)\left[p_{0} \bar{P}(u+1-k)+q_{0} \bar{P}(u-k)\right] \\
& +\frac{p q_{1}}{p_{0} p_{1} q_{k=u+1}} \sum^{\infty}\left[p_{0} \bar{P}(k)+q_{0} \bar{P}(k-1)\right] \\
& +\frac{p}{p_{0} q_{k=u+1}} \sum_{k=1}^{\infty}\left[p_{0} \bar{P}(k+1)+q_{0} \bar{P}(k)\right], \quad \forall u \geq b,
\end{aligned}
$$

and the following linear equations: when $b=0$

$$
\psi(0)=\frac{p}{p_{0} p_{1} q}\left(\mu-p_{0} p_{1}\right),
$$

or, when $b \geq 1$,

$$
p_{1} q \psi(0)-q_{0} \psi(b-1)=p\left(\mu-p_{1}\right),
$$

$q p_{1} \psi(u+1)+\left(q q_{1}+p p_{1} p(1)-1\right) \psi(u)$

$$
\begin{aligned}
& +p q_{1} \sum_{k=0}^{u-1} \psi(k) p(u-k) \\
& +p p_{1} \sum_{k=0}^{u-1} \psi(k) p(u+1-k) \\
& =f(u+1), \quad(u=0,1,2, \ldots, b-1) .
\end{aligned}
$$


Example 3. Letting $w\left(x_{1}, x_{2}\right)=I\left(x_{2} \leq y\right)(y=1,2, \ldots)$ and $v=1$, then

$$
m(u)=\operatorname{Pr}\left(\left|U_{T}\right| \leq y, T<\infty\right)=H(u, y)
$$

is the distribution function of the deficit at ruin. In this case,

$$
\begin{aligned}
f(u+1)= & -p q_{1}[P(y+u)-P(u)] \\
& -p p_{1}[P(y+u+1)-P(u+1)], \\
\delta & =p \sum_{k=1}^{y} \bar{P}(k)-p q_{1} \bar{P}(y) .
\end{aligned}
$$

Thus, $H(u, y)$ satisfies the following recursive formula $(\forall u \geq$ b):

$$
\begin{aligned}
H & (u+1, y) \\
= & \frac{1-q q_{1} p_{0}-q p_{1} q_{0}}{q p_{1} p_{0}} H(u, y) \\
& -\frac{q_{1} q_{0}}{p_{1} p_{0}} H(u-1, y) \\
& -\frac{p q_{1}}{q p_{1} p_{0}} \sum_{k=0}^{u-1} H(k, y)\left[p_{0} p(u-k)+q_{0} p(u-1-k)\right] \\
& -\frac{p q_{1}}{q p_{1} p_{0}} \sum_{k=1}^{y}\left[p_{0} p(u+k)+q_{0} p(u+k-1)\right] \\
& -\frac{p}{q p_{0}} \sum_{k=0}^{u} H(k, y)\left[p_{0} p(u+1-k)+q_{0} p(u-k)\right] \\
& -\frac{p}{q p_{0}} \sum_{k=1}^{y}\left[p_{0} p(u+k+1)+q_{0} p(u+k)\right]
\end{aligned}
$$

when $b \geq 1$,

$$
\begin{gathered}
p_{1} q H(0, y)-q_{0} H(b-1, y)=p \sum_{k=1}^{y} \bar{P}(k)-p q_{1} \bar{P}(y) \\
q p_{1} H(u+1, y)+\left(q q_{1}+p p_{1} p(1)-1\right) H(u, y) \\
+p q_{1} \sum_{k=0}^{u-1} H(k, y) p(u-k) \\
\quad+p p_{1} \sum_{k=0}^{u-1} H(k, y) p(u+1-k) \\
=f(u+1), \quad(u=0,1,2, \ldots, b-1) .
\end{gathered}
$$

Example 4. Letting $w\left(x_{1}, x_{2}\right)=r^{x_{2}}(0 \leq r<1)$ and $v=1$, then

$$
m(u)=E\left[r^{\left|U_{T}\right|} I(T<\infty)\right]=\widehat{H}(u, r)
$$

is the generating function of the deficit at ruin. In this case,

$$
f(u+1)=-p q_{1} \sum_{k=1}^{\infty} r^{k} p(u+k)-p p_{1} \sum_{k=1}^{\infty} r^{k} p(u+k+1) \text {. }
$$

For all $u \geq b$, we have

$$
\begin{aligned}
\widehat{H}(u & +1, r) \\
= & \frac{1-q q_{1} p_{0}-q p_{1} q_{0}}{q p_{1} p_{0}} \widehat{H}(u, r) \\
& -\frac{q_{1} q_{0}}{p_{1} p_{0}} \widehat{H}(u-1, r) \\
& -\frac{p q_{1}}{q p_{1} p_{0}} \sum_{k=0}^{u-1} \widehat{H}(k, r)\left[p_{0} p(u-k)+q_{0} p(u-1-k)\right] \\
& -\frac{p q_{1}}{q p_{1} p_{0}} \sum_{k=1}^{\infty}\left[p_{0} p(u+k)+q_{0} p(u+k-1)\right] \\
& -\frac{p}{q p_{0}} \sum_{k=0}^{u} \widehat{H}(k, r)\left[p_{0} p(u+1-k)+q_{0} p(u-k)\right] \\
& -\frac{p}{q p_{0}} \sum_{k=1}^{\infty}\left[p_{0} p(u+k+1)+q_{0} p(u+k)\right] .
\end{aligned}
$$

When $b \geq 1$,

$$
\begin{gathered}
p_{1} q \widehat{H}(0, r)-q_{0} \widehat{H}(b-1, r)=\frac{r p-G_{X}(r)\left(p p_{1}+r p q_{1}\right)}{1-r} \\
q p_{1} \widehat{H}(u+1, r)+\left(q q_{1}+p p_{1} p(1)-1\right) \widehat{H}(u, r) \\
\quad+p q_{1} \sum_{k=0}^{u-1} \widehat{H}(k, r) p(u-k) \\
\quad+p p_{1} \sum_{k=0}^{u-1} \widehat{H}(k, r) p(u+1-k) \\
=f(u+1), \quad(u=0,1,2, \ldots, b-1) .
\end{gathered}
$$

4.2. Numerical Illustration. In the following example, we investigate the impact of the inclusion of the dividend payments, claim, and stochastic premium income on ruin probability. We suppose that the claim amounts $X_{i}$ have a zero-truncated geometric distribution with mean $\mu=6$ and the probability function $p(k)=1 / 6 \times(1-1 / 6)^{k-1}, i=$ $1,2,3, \ldots$ Let $b=5$. We aim to compute the ruin probabilities with different values of the $q_{0}, p_{1}$, and $p$. The results are given in Tables 1 and 2.

As expected, the tables show that ruin probability increases as $q_{0}$ and $p$ increase while ruin probability decreases as $p_{1}$ increases. It is worth mentioning that when the initial surplus $u$ is large enough (e.g., $u=100$ ), the difference of ruin probability is very little. This illustrates the importance of enhancing the initial capital. Furthermore, in order to more vividly reflect the influence of the parameters on ruin probability we provide Figures 1, 2, and 3, which show the impact of $p_{1}, q_{0}$ and $p$ on ruin probability, respectively. 
TABLE 1: Exact values for $\psi(u)$.

\begin{tabular}{|c|c|c|c|c|c|c|}
\hline \multicolumn{7}{|c|}{$p=0.08$} \\
\hline \multirow[t]{2}{*}{$u$} & \multicolumn{2}{|c|}{$q_{0}=0$} & \multicolumn{2}{|c|}{$q_{0}=0.1$} & \multicolumn{2}{|c|}{$q_{0}=0.15$} \\
\hline & $p_{1}=0.9$ & $p_{1}=0.95$ & $p_{1}=0.9$ & $p_{1}=0.95$ & $p_{1}=0.9$ & $p_{1}=0.95$ \\
\hline 0 & 0.4928 & 0.4622 & 0.5423 & 0.5047 & 0.5746 & 0.5319 \\
\hline 1 & 0.4511 & 0.4208 & 0.5047 & 0.4666 & 0.5396 & 0.4958 \\
\hline 2 & 0.4130 & 0.3831 & 0.4703 & 0.4319 & 0.5076 & 0.4630 \\
\hline 3 & 0.3781 & 0.3488 & 0.4388 & 0.4002 & 0.4784 & 0.4331 \\
\hline 4 & 0.3461 & 0.3175 & 0.4099 & 0.3714 & 0.4515 & 0.4059 \\
\hline 5 & 0.3168 & 0.2891 & 0.3835 & 0.3452 & 0.4270 & 0.3812 \\
\hline 6 & 0.2900 & 0.2632 & 0.3558 & 0.3180 & 0.3993 & 0.3537 \\
\hline 7 & 0.2655 & 0.2396 & 0.3300 & 0.2929 & 0.3733 & 0.3281 \\
\hline 8 & 0.2430 & 0.2182 & 0.3062 & 0.2699 & 0.3490 & 0.3043 \\
\hline 9 & 0.2225 & 0.1986 & 0.2840 & 0.2486 & 0.3263 & 0.2823 \\
\hline 10 & 0.2037 & 0.1808 & 0.2634 & 0.2290 & 0.3051 & 0.2619 \\
\hline 20 & 0.0842 & 0.0708 & 0.1243 & 0.1007 & 0.1557 & 0.1236 \\
\hline 30 & 0.0347 & 0.0277 & 0.0596 & 0.0443 & 0.0795 & 0.0584 \\
\hline 40 & 0.0143 & 0.0109 & 0.0276 & 0.0195 & 0.0406 & 0.0276 \\
\hline 50 & 0.0059 & 0.0043 & 0.0130 & 0.0085 & 0.0207 & 0.0131 \\
\hline 60 & 0.0024 & 0.0018 & 0.0061 & 0.0037 & 0.0106 & 0.0062 \\
\hline 70 & 0.0009 & 0.0007 & 0.0029 & 0.0016 & 0.0054 & 0.0030 \\
\hline 80 & 0.0003 & 0.0004 & 0.0023 & 0.0007 & 0.0028 & 0.0015 \\
\hline 90 & 0.0001 & 0.0002 & 0.0006 & 0.0003 & 0.0014 & 0.0008 \\
\hline 100 & 0.0000 & 0.0001 & 0.0003 & 0.0001 & 0.0008 & 0.0005 \\
\hline
\end{tabular}

TABLE 2: Exact values for $\psi(u)$.

\begin{tabular}{|c|c|c|c|c|c|c|}
\hline \multicolumn{7}{|c|}{$p=0.095$} \\
\hline \multirow[t]{2}{*}{$u$} & \multicolumn{2}{|c|}{$q_{0}=0$} & \multicolumn{2}{|c|}{$q_{0}=0.1$} & \multicolumn{2}{|c|}{$q_{0}=0.15$} \\
\hline & $p_{1}=0.9$ & $p_{1}=0.95$ & $p_{1}=0.9$ & $p_{1}=0.95$ & $p_{1}=0.9$ & $p_{1}=0.95$ \\
\hline 0 & 0.5948 & 0.5580 & 0.6611 & 0.6146 & 0.7053 & 0.6514 \\
\hline 1 & 0.5547 & 0.5169 & 0.6275 & 0.5787 & 0.6761 & 0.6190 \\
\hline 2 & 0.5172 & 0.4788 & 0.5962 & 0.5455 & 0.6488 & 0.5890 \\
\hline 3 & 0.4823 & 0.4436 & 0.5670 & 0.5148 & 0.6234 & 0.5612 \\
\hline 4 & 0.4497 & 0.4109 & 0.5397 & 0.4863 & 0.5997 & 0.5354 \\
\hline 5 & 0.4194 & 0.3806 & 0.5143 & 0.4599 & 0.5777 & 0.5115 \\
\hline 6 & 0.3911 & 0.3526 & 0.4871 & 0.4320 & 0.5523 & 0.4845 \\
\hline 7 & 0.3647 & 0.3266 & 0.4614 & 0.4059 & 0.5280 & 0.4589 \\
\hline 8 & 0.3401 & 0.3025 & 0.4370 & 0.3831 & 0.5047 & 0.4346 \\
\hline 9 & 0.3171 & 0.2802 & 0.4139 & 0.3581 & 0.4825 & 0.4116 \\
\hline 10 & 0.2957 & 0.2596 & 0.3920 & 0.3364 & 0.4613 & 0.3898 \\
\hline 20 & 0.1470 & 0.1208 & 0.2277 & 0.1801 & 0.2940 & 0.2264 \\
\hline 30 & 0.0731 & 0.0562 & 0.1323 & 0.0964 & 0.1874 & 0.1315 \\
\hline 40 & 0.0364 & 0.0261 & 0.0768 & 0.0516 & 0.1195 & 0.0763 \\
\hline 50 & 0.0181 & 0.0121 & 0.0446 & 0.0276 & 0.0762 & 0.0443 \\
\hline 60 & 0.0091 & 0.0056 & 0.0259 & 0.0148 & 0.0487 & 0.0257 \\
\hline 70 & 0.0046 & 0.0026 & 0.0150 & 0.0080 & 0.0311 & 0.0149 \\
\hline 80 & 0.0023 & 0.0012 & 0.0087 & 0.0043 & 0.0199 & 0.0086 \\
\hline 90 & 0.0012 & 0.0005 & 0.0050 & 0.0023 & 0.0128 & 0.0050 \\
\hline 100 & 0.0007 & 0.0003 & 0.0028 & 0.0012 & 0.0082 & 0.0028 \\
\hline
\end{tabular}




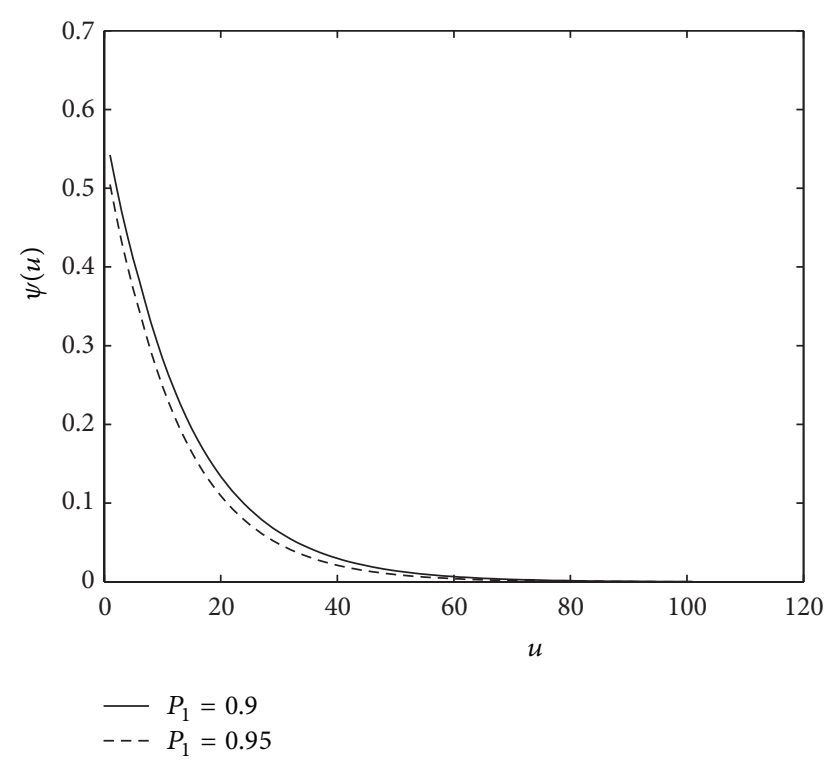

FIGURE 1: The impact of $p_{1}$ on ruin probability.

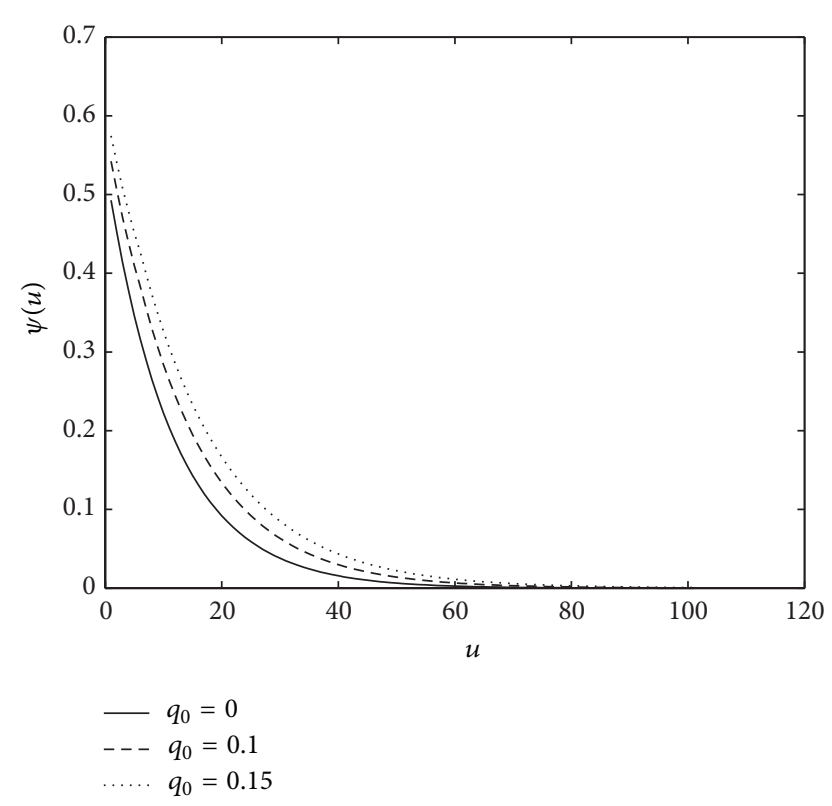

FIGURE 2: The impact of $q_{0}$ on ruin probability.

\section{Acknowledgments}

The author thanks the three anonymous referees for the thoughtful comments and suggestions that greatly improved the presentation of this paper. This work was supported by National Natural Science Foundation of China (Grant no. 11171187 and Grant no. 10921101), National Basic Research Program of China (973 Program, Grant no. 2007CB814906), Natural Science Foundation of Shandong Province (Grant no. ZR2012AQ013 and Grant no. ZR2010GL013), and Humanities and Social Sciences Project of the Ministry Education of China (Grant no. 10YJC630092 and Grant no. 09YJC910004).

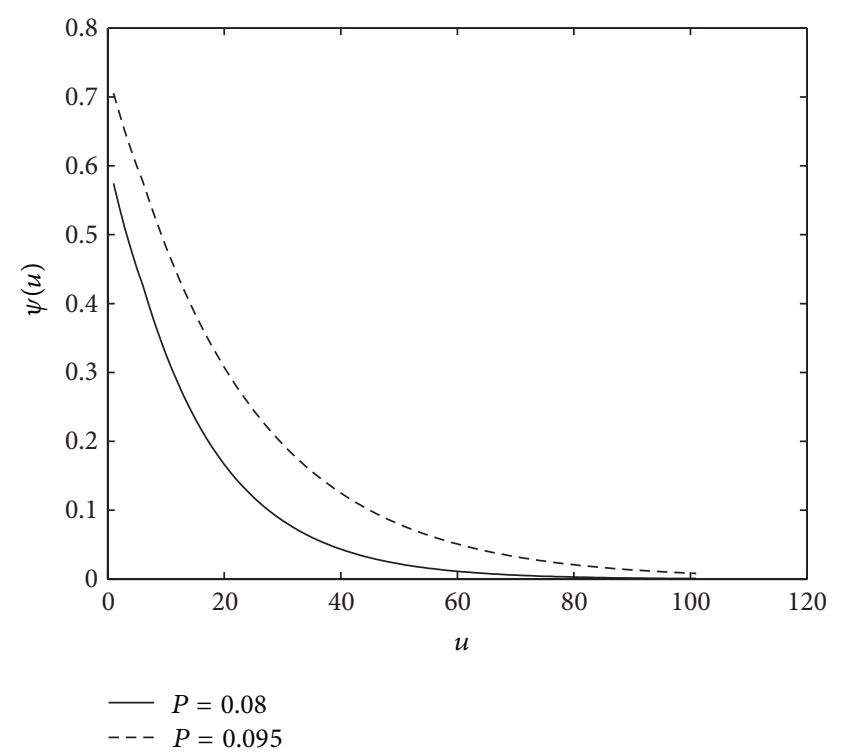

FIGURE 3: The impact of $p$ on ruin probability.

\section{References}

[1] H. U. Gerber, "Mathematical fun with the compound binomial process," Astin Bulletin, vol. 18, no. 2, pp. 161-168, 1988.

[2] E. S. W. Shiu, "The probability of eventual ruin in the compound binomial model," Astin Bulletin, vol. 19, no. 2, pp. 179-190, 1989.

[3] G. E. Willmot, "Ruin probabilities in the compound binomial model," Insurance: Mathematics \& Economics, vol. 12, no. 2, pp. 133-142, 1993.

[4] D. C. M. Dickson, "Some comments on the compound binomial model," Astin Bulletin, vol. 24, no. 1, pp. 33-45, 1994.

[5] H. U. Gerber and E. S. W. Shiu, "On the time value of ruin," North American Actuarial Journal, vol. 2, no. 1, pp. 48-78, 1998.

[6] S. Cheng, H. U. Gerber, and E. S. W. Shiu, "Discounted probabilities and ruin theory in the compound binomial model," Insurance: Mathematics \& Economics, vol. 26, no. 2-3, pp. 239250, 2000.

[7] X. S. Lin, G. E. Willmot, and S. Drekic, "The classical risk model with a constant dividend barrier: analysis of the GerberShiu discounted penalty function," Insurance: Mathematics \& Economics, vol. 33, no. 3, pp. 551-566, 2003.

[8] G. Wang and R. Wu, "The expected discounted penalty function for the perturbed compound Poisson risk process with constant interest," Insurance: Mathematics \& Economics, vol. 42, no. 1, pp. 59-64, 2008.

[9] W. Wang, R. Ming, and Y. Hu, "On the expected discounted penalty function for risk process with tax," Statistics \& Probability Letters, vol. 81, no. 4, pp. 489-501, 2011.

[10] G. E. Willmot and J.-K. Woo, "On the analysis of a general class of dependent risk processes," Insurance: Mathematics \& Economics, vol. 51, no. 1, pp. 134-141, 2012.

[11] J. Y. Tan and X. Q. Yang, "The compound binomial model with randomized decisions on paying dividends," Insurance: Mathematics \& Economics, vol. 39, no. 1, pp. 1-18, 2006.

[12] D. Landriault, "Randomized dividends in the compound binomial model with a general premium rate," Scandinavian Actuarial Journal, no. 1, pp. 1-15, 2008. 
[13] S.-Z. Fang, P.-C. Zhao, and C.-M. Zhang, "The expected discounted penalty function at ruin of the discrete risk model with random income," Mathematica Applicata, vol. 21, no. 4, pp. 771-777, 2008.

[14] S. M. Li, "The moments of the present value of total dividends in the compound binomial model under a constant dividend barrier and stochastic interest rates," Australian Actuarial Journal, vol. 14, no. 2, pp. 175-192, 2008.

[15] K.-C. Yuen and J. Guo, "Some results on the compound Markov binomial model," Scandinavian Actuarial Journal, no. 3, pp. 129140, 2006.

[16] G. Liu and J. Zhao, "Joint distributions of some actuarial random vectors in the compound binomial model," Insurance: Mathematics \& Economics, vol. 40, no. 1, pp. 95-103, 2007.

[17] Y.-B. Yu, L.-X. Zhang, and Y. Zhang, "Joint and supremum distributions in the compound binomial model with Markovian environment," Applied Mathematics, vol. 26, no. 3, pp. 265-279, 2011.

[18] Y. Y. Hao and H. Yang, "On a compound Poisson risk model with delayed claims and random incomes," Applied Mathematics and Computation, vol. 217, no. 24, pp. 10195-10204, 2011.

[19] J. Y. Tan and X. Q. Yang, "The compound binomial model with a constant dividend barrier and periodically paid dividends," Journal of Systems Science \& Complexity, vol. 25, no. 1, pp. 167177, 2012.

[20] Z. H. Bao and H. Liu, "The compound binomial risk model with delayed claims and random income," Mathematical and Computer Modelling, vol. 55, no. 3-4, pp. 1315-1323, 2012. 


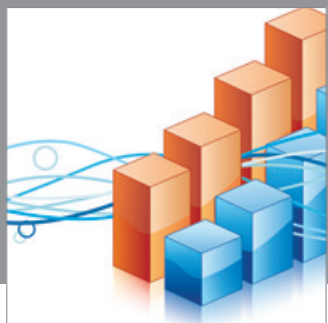

Advances in

Operations Research

mansans

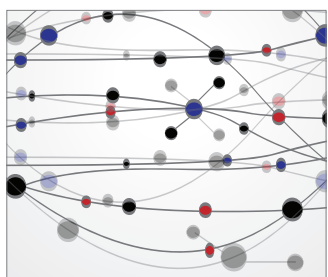

The Scientific World Journal
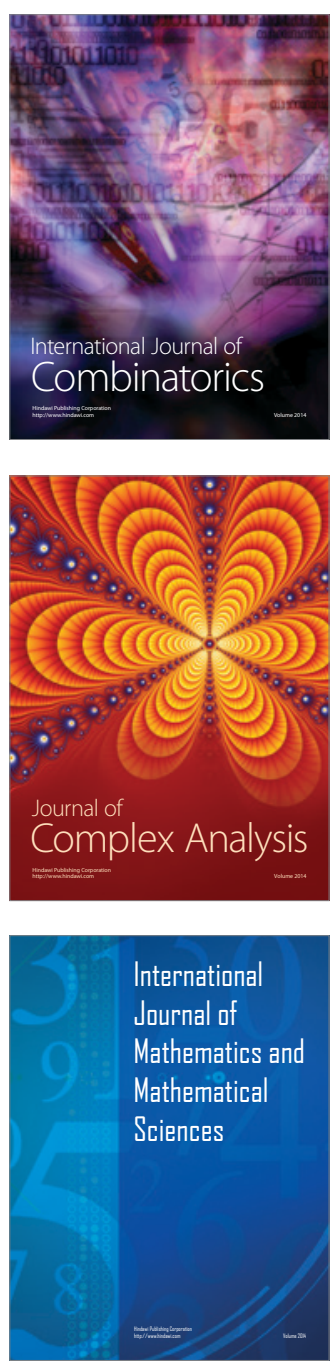
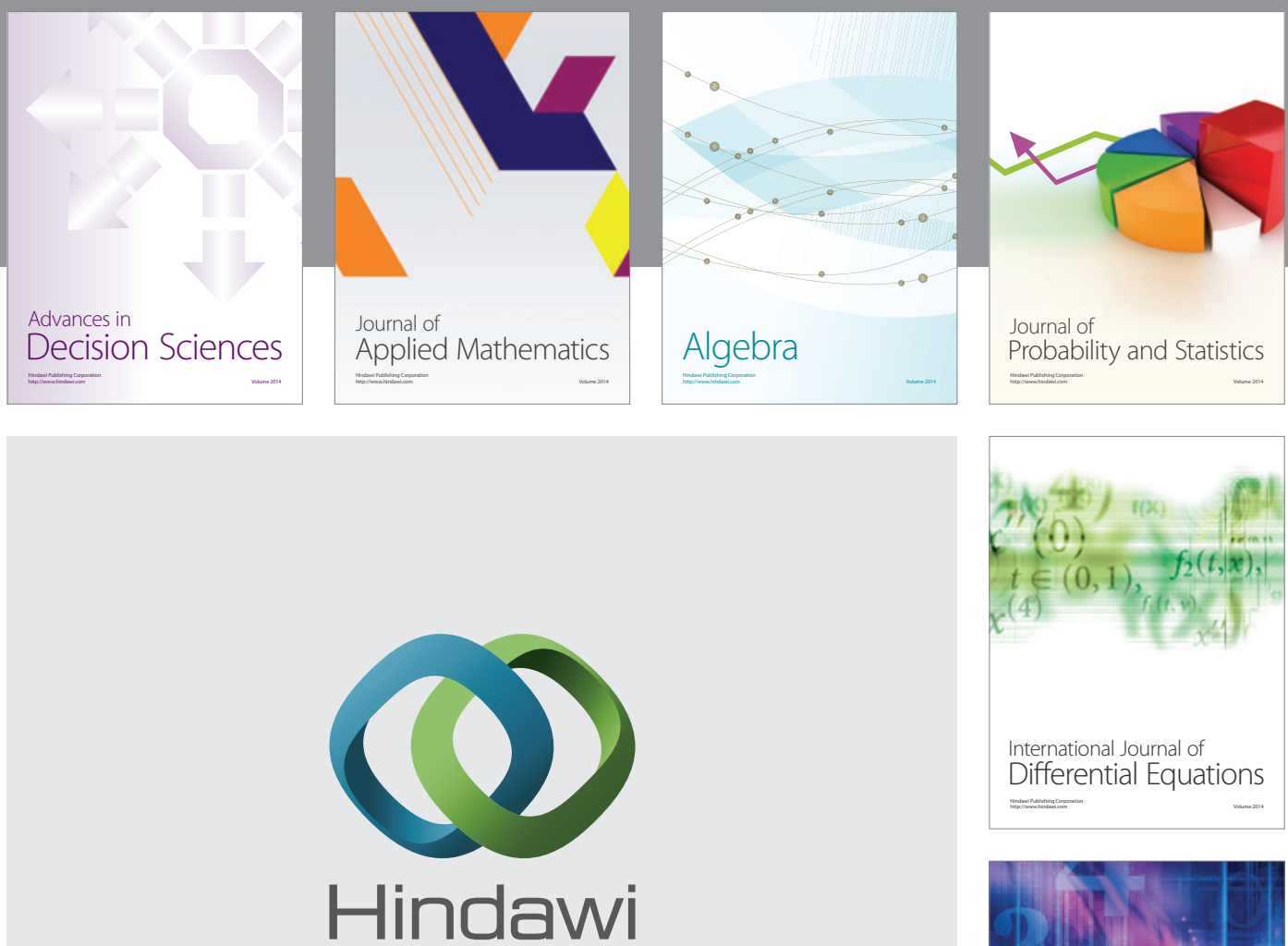

Submit your manuscripts at http://www.hindawi.com
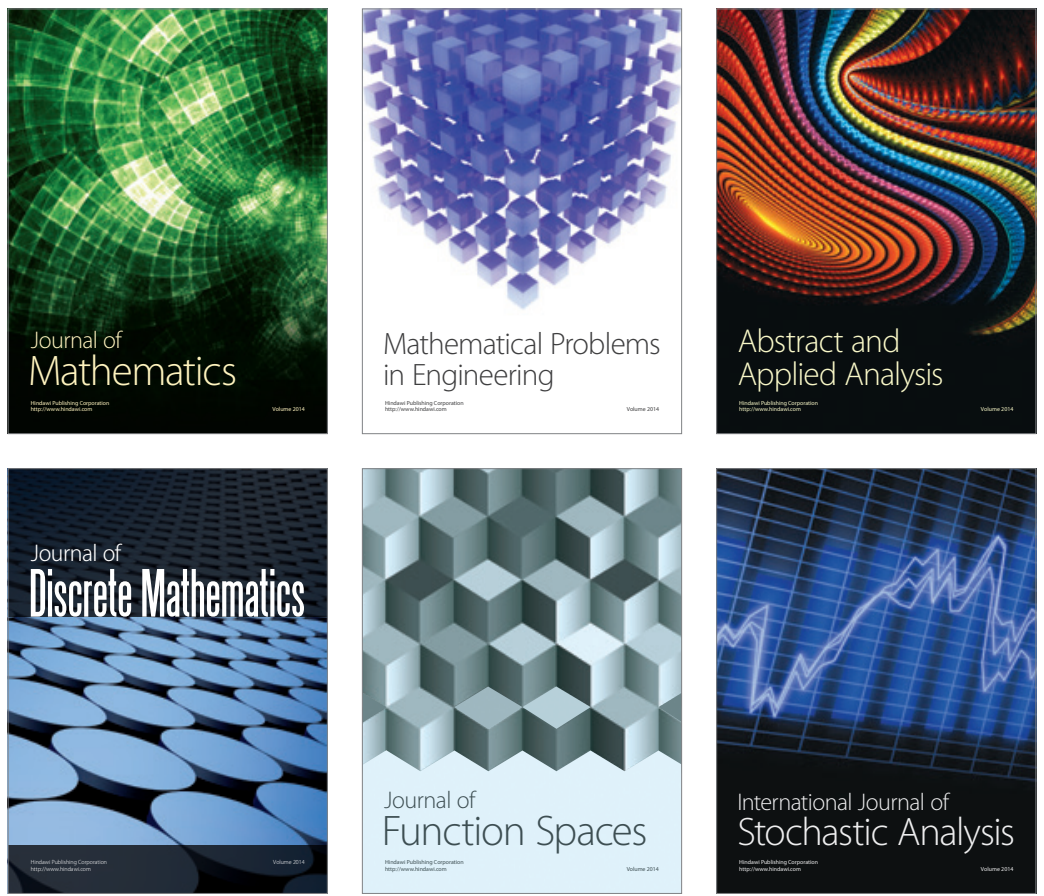

Journal of

Function Spaces

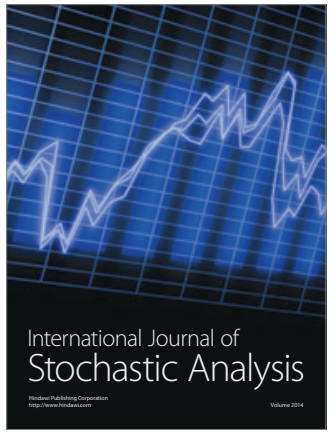

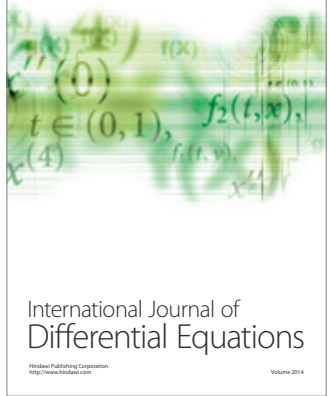
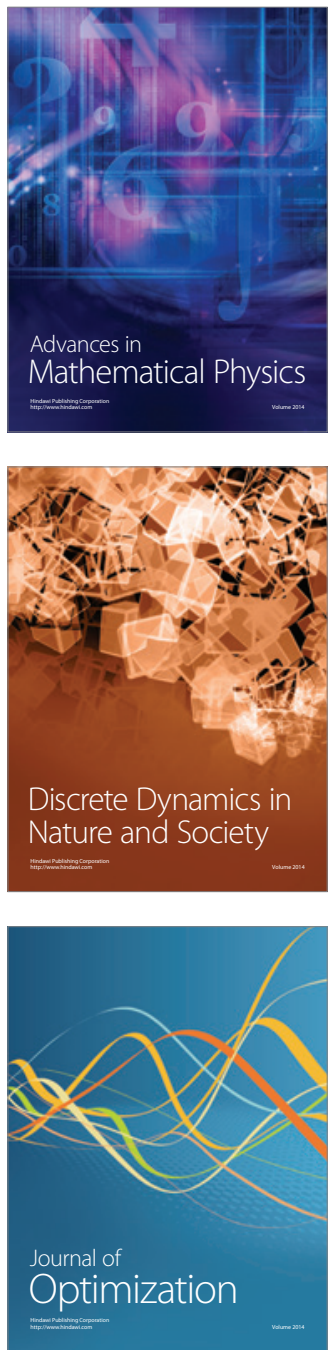